\title{
Anesthetic management of laparoscopic pheochromocytoma excision in a patient with a Fontan circulation
} -a case report-

\author{
Hyung-Chul Lee, Karam Nam, Ji-Hyun Lee, Yong-Hee Park, Hee-Soo Kim, Chong-Sung Kim, and \\ Jin-Tae Kim
}

Department of Anesthesiology and Pain Medicine, Seoul National University Hospital, Seoul, Korea

An 18-year-old male with a Fontan circulation underwent excision of a pheochromocytoma after conversion from laparoscopic surgery. The pneumoperitoneum established for laparoscopic surgery may have adverse effects on the Fontan circulation, because it increases the intra-abdominal pressure (IAP), intra-thoracic pressure, pulmonary vascular resistance, and systemic vascular resistance (SVR), and decreases cardiac preload and cardiac output. Meticulous monitoring is also required during carbon dioxide exsufflation, because a rapid decrease in IAP can provoke hemodynamic deterioration by decreasing venous return and SVR. Furthermore, catecholamines released by the pheochromocytoma can worsen the hemodynamic status of Fontan circulation during surgery. Therefore, sophisticated intraoperative anesthetic care is required during laparoscopic pheochromocytoma excision in patients with a Fontan circulation. (Korean J Anesthesiol 2014; 66: 252-255)

Key Words: Fontan procedure, General anesthesia, Laparoscopic surgery, Pheochromocytoma.

We experienced a rare anesthetic case of laparoscopic pheochromocytoma excision that was converted to laparotomy in an 18-year-old male with a Fontan circulation. The adverse effects associated with pneumoperitoneum established for laparoscopy in a patient with a single ventricle, as in a Fontan circulation, are rarely documented. In addition, catecholamines secreted from a pheochromocytoma can lead to severe hemodynamic instability. However, there is no case report of laparoscopic surgery for a pheochromocytoma in a patient with a Fontan circulation.

\section{Case Report}

An 18-year-old 170-cm-tall male weighing $51 \mathrm{~kg}$ was admitted via the emergency room complaining of palpitations, dyspnea, and cyanosis. The patient was born with complex congenital cardiac anomalies, consisting of a single ventricle (right ventricle type), a complete endocardial cushion defect, corrected transposition of the great arteries, right isomerism, and a supracardiac type total anomalous pulmonary venous return. The

Received: December 11, 2012. Revised: 1st, January 18, 2013; 2nd, February 15, 2013. Accepted: February 15, 2013.

Corresponding author: Jin-Tae Kim, M.D., Ph.D., Department of Anesthesiology and Pain Medicine, Seoul National University Hospital, 101, Daehak-ro, Jongno-gu, Seoul 110-744, Korea. Tel: 82-2-2072-3580, Fax: 82-2-747-5639, E-mail: kimjintae73@dreamwiz.com

(C) This is an open-access article distributed under the terms of the Creative Commons Attribution Non-Commercial License (http:// creativecommons.org/licenses/by-nc/3.0/), which permits unrestricted non-commercial use, distribution, and reproduction in any medium, provided the original work is properly cited. 
patient underwent correction of the total anomalous pulmonary venous return and bidirectional cavo-pulmonary shunt when he was 11 months old, and a Fontan procedure at age 3 years. The patient was taking aspirin (200 mg), digoxin (0.25 mg), enalapril (5 mg) and, atenolol (12.5 mg) daily. The initial vital signs were a systolic blood pressure (SBP) of $126 \mathrm{mmHg}$ and heart rate (HR) of 116 beats/min. Arterial blood sampling was obtained on room air, and showed hypoxemia $\left(\mathrm{PaO}_{2} 58 \mathrm{mmHg}\right)$. The patient also had compensatory polycythemia (hemoglobin $15.8 \mathrm{~g} / \mathrm{dl}$ ) secondary to chronic hypoxemia. The last cardiac catheterization was performed when the patient was 12 years old, and it showed only mild atrio-ventricular valve regurgitation (AVVR). The mean pressure of the Fontan pathway was $13 \mathrm{mmHg}$. Laboratory testing revealed increased serum $(3,533 \mathrm{pg} / \mathrm{ml}$; normal $<1,050 \mathrm{pg} /$ $\mathrm{ml})$ and urine $(768.2 \mu \mathrm{g} /$ day; normal $<97 \mu \mathrm{g} /$ day $)$ norepinephrine and urine vanillylmandelic acid ( $8.8 \mathrm{mg}$ /day; normal 2-7 $\mathrm{mg}$ /day) levels. Computed tomography showed a $4 \mathrm{~cm}$ mass in the left para-aortic area above the left kidney. With an impression of pheochromocytoma, the patient was scheduled for laparoscopic excision of the tumor. Phenoxybenzamine (10 mg) was administered daily for 2 weeks before the surgery. Preoperative electrocardiography showed supraventricular tachycardia and $24 \mathrm{~h}$ Holter monitoring showed no significant cardiac arrhythmia except intermittent junctional rhythm. Echocardiography revealed mild AVVR. The estimated ejection fraction of the ventricle was approximately $50 \%$ and the ventricular wall motion was normal. There was no obvious stenosis within the Fontan pathway or the scanned parts of pulmonary vessels.

After being administered intravenous midazolam $(1.5 \mathrm{mg})$, the patient was brought to the operating room. The initial SBP and HR there were $144 \mathrm{mmHg}$ and 94 beats/min, respectively. Anesthesia was induced with intravenous midazolam (2.5 mg), etomidate $(10 \mathrm{mg})$, remifentanil $(40 \mu \mathrm{g})$, and rocuronium $(50 \mathrm{mg})$. The trachea was intubated and anesthesia was maintained with sevoflurane and 50\% oxygen in $2 \mathrm{~L} / \mathrm{min}$ fresh gas. In addition, intravenous remifentanil was infused continuously during the surgery. The right radial artery was cannulated for continuous arterial blood pressure monitoring. A central venous catheter was placed in the right internal jugular vein, and continuous central venous pressure (CVP) monitoring was started. The initial CVP was $15 \mathrm{mmHg}$. A transesophageal echocardiography (TEE) probe was inserted smoothly and the echocardiographic evaluation revealed normal ventricular function with mild AVVR. After achieving hemodynamic stability, a continuous infusion of milrinone $(0.5 \mu \mathrm{g} / \mathrm{kg} / \mathrm{min})$ and magnesium sulfate $\left(\mathrm{MgSO}_{4}\right)(1 \mathrm{~g} / \mathrm{h})$ was started after a 1 -g loading dose of $\mathrm{MgSO}_{4}$ to prevent severe pulmonary hypertension and maintain a stable blood pressure during the surgery.

Surgery was started, and intra-abdominal $\mathrm{CO}_{2}$ insufflation was begun for the laparoscopic procedure. After insufflation, the
CVP was increased to $28 \mathrm{mmHg}$ and the SBP rose from 90 to $128 \mathrm{mmHg}$ (Fig. 1). Simultaneously, the peak inspiratory pressure increased from 17 to $24 \mathrm{cmH}_{2} \mathrm{O}$. The arterial oxygen saturation decreased from 100 to $97 \%$, and the arterial oxygen partial pressure dropped from 196 to $93 \mathrm{mmHg}$. The intra-abdominal pressure (IAP) was maintained between 13 and $15 \mathrm{mmHg}$. The inspired oxygen fraction was transiently set to 1.0 , and an immediate TEE evaluation showed preserved ventricular contractility without aggravation of the mild AVVR. To prevent abrupt arterial hypertension, a continuous infusion of sodium nitroprusside (SNP) was started $(0.5 \mu \mathrm{g} / \mathrm{kg} / \mathrm{min})$ and titrated up to $1.5 \mu \mathrm{g} /$ $\mathrm{kg} / \mathrm{min}$. However, four further sudden increases in SBP over 160 $\mathrm{mmHg}$, up to $194 \mathrm{mmHg}$, occurred during the dissection and approach to the tumor; these were controlled by intravenous bolus injections of SNP (30 or $40 \mu \mathrm{g}$ ) and remifentanil (40 or 50 $\mu \mathrm{g})$. No significant cardiac arrhythmias were detected, except sinus tachycardia during the dissection.

Approximately $2 \mathrm{~h}$ after establishing pneumoperitoneum, the surgeon decided to convert from laparoscopic surgery to a laparotomy, suspecting aortic invasion of the tumor. After exsufflation, the CVP decreased from 18 to $10 \mathrm{mmHg}$ and the SBP dropped from 120 to $70 \mathrm{mmHg}$. Subsequent TEE showed decreased ventricular volume with preserved contractility. After fluid resuscitation and continuous infusion of dopamine $(5 \mu \mathrm{g} / \mathrm{kg} / \mathrm{min})$ and norepinephrine $(0.02 \mu \mathrm{g} / \mathrm{kg} / \mathrm{min})$ for approximately $7 \mathrm{~min}$, the SBP recovered and was maintained at $100-120 \mathrm{mmHg}$. Arterial blood gas analysis showed that the oxygen partial pressure had increased to $157 \mathrm{mmHg}$. Subsequently, there were several abrupt

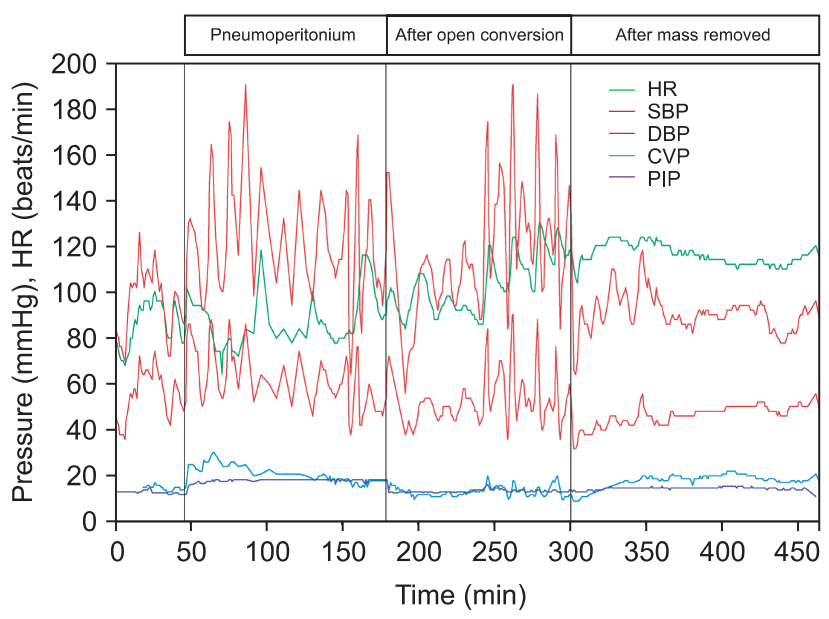

Fig. 1. Hemodynamic changes during the operation. There are more fluctuations of hemodynamic variables during laparoscopy, as compared to laparotomy. There are abrupt increases in SBP, CVP, and PIP at the beginning of pneumoperitoneum. Immediately after exsufflation, acute decreases in SBP, CVP, and PIP are observed. HR: heart rate (beats/ $\mathrm{min})$, SBP: systolic arterial blood pressure $(\mathrm{mmHg}), \mathrm{DBP}$ : diastolic arterial blood pressure $(\mathrm{mmHg}), \mathrm{CVP}$ : central venous pressure $(\mathrm{mmHg})$, PIP: peak inspiratory pressure ( $\mathrm{mmHg}$ ). 
increases in SBP up to $190 \mathrm{mmHg}$ until the tumor was excised; these were well controlled with bolus injections of SNP and remifentanil.

The tumor was removed approximately 260 min after beginning the surgery. After removing the tumor, the $\mathrm{MgSO}_{4}$ was discontinued and the milrinone was tapered $(0.25 \mu \mathrm{g} / \mathrm{kg} / \mathrm{min})$. Continuous infusion of dopamine $(5 \mu \mathrm{g} / \mathrm{kg} / \mathrm{min})$ and norepinephrine $(0.04 \mu \mathrm{g} / \mathrm{kg} / \mathrm{min})$ was initiated. The infusion rates were tapered to 3 and $0.02 \mu \mathrm{g} / \mathrm{kg} / \mathrm{min}$, respectively, before the end of surgery. The duration of the surgery and anesthesia was 490 min. The patient was transferred to the surgical intensive care unit and extubated $12 \mathrm{~h}$ later. Intravenous patient-controlled analgesia with fentanyl was used to manage postoperative pain, which was relatively well controlled. All inotropics were discontinued on the third postoperative day. Subsequently, the patient was moved to a general ward and discharged uneventfully on the $13^{\text {th }}$ postoperative day.

\section{Discussion}

The Fontan procedure was introduced in 1971 as a method of establishing a physiological circulation in patients born with congenital tricuspid atresia [1]. In the Fontan circulation and its modifications, the systemic venous return is not drained to the right heart, but to the pulmonary arteries directly, so that the systemic venous return bypasses the pumping heart.

A pheochromocytoma is catecholamine-secreting tumor originating from chromaffin cells in the adrenal gland, and usually presents with palpitations, syncope, anxiety, hypertension, and hypergylcemia [2]. The perioperative management and anesthetic care of patients with pheochromocytoma are relatively well documented $[3,4]$. Furthermore, there are many case reports of successful anesthetic care in patients with pheochromocytoma. However, anesthesia case reports of pheochromocytoma excision in patients with a single ventricle, such as a Fontan circulation, are very rare [5-7]. In addition, no case of anesthesia for laparoscopic pheochromocytoma excision in a patient with a Fontan circulation has been reported.

In a Fontan circulation, the pulmonary blood flow is determined passively by the CVP, left atrial pressure (LAP), left ventricular end-diastolic pressure (LVEDP), and pulmonary vascular resistance (PVR) [8]. A sufficiently high CVP and low LAP, LVEDP, and PVR are required to maintain adequate pulmonary blood flow, because there is no ventricle for the pulmonary circulation. Accordingly, we should avoid conditions that result in a low CVP, high LAP or LVEDP, and high PVR being created.

The pneumoperitoneum for laparoscopy can increase the intra-abdominal, intra-thoracic, and airway pressures, which increase the PVR. Moreover, hypercapnia or atelectasis that develops during laparoscopy can worsen the increased PVR.
Therefore, pneumoperitoneum can provoke profound pulmonary hypoperfusion and severe hypoxia more easily in patients with a Fontan circulation, as in our case. In addition, the catecholamines secreted by a pheochromocytoma can decrease $\mathrm{CO}$, which is associated with an increase in SVR. Vasodilatory agents might be considered in these situations. Nitroglycerin or SNP can play a crucial role in controlling a high SVR, and milrinone is effective at lowering the PVR. We started a milrinone infusion in our patient before the pneumoperitoneum to control low $\mathrm{CO}$ that developed after the pneumoperitoneum. $\mathrm{MgSO}_{4}$ is also effective at producing hemodynamic stability due to its anti-hypertensive and anti-arrhythmic properties in these patients $[9,10]$. Note that $\mathrm{CO}_{2}$ exsufflation is as challenging as insufflation to anesthesiologists in these patients. While the high IAP occurring during pneumoperitoneum decreases the blood volume capacitance of the splanchnic vasculature, an abrupt decrease in IAP can cause significant volume pooling in the capacitance vessels. Along with an acute decrease in SVR, the decreased venous return caused by splanchnic venous pooling can provoke severe hypotension. Sufficient fluid resuscitation and temporary vasopressor infusion can help, as in our case.

A thorough preoperative evaluation is as important as careful intraoperative anesthetic management in patients with a Fontan circulation. The medical history should include exercise tolerance and recent illnesses, such as respiratory tract infections, which can potentially affect the airway resistance or PVR [11]. Laboratory tests, including the coagulation profile and liver function tests, should also be reviewed since liver function abnormalities and thromboembolism are well-known complications in Fontan patients [12,13]. The systemic evaluation should focus on the anatomical and functional integrity of the Fontan circulation, and echocardiography or cardiac catheterization might be helpful. The patency of the Fontan pathway and pulmonary arterial pressure can be measured by echocardiography or cardiac catheterization. Valvular function, including the aortic and atrioventricular valves, can also be assessed by echocardiography. Ventricular function also has to be evaluated. An electrocardiogram can play an important role in detecting hidden arrhythmias, which are much more hazardous in these patients. In addition, detailed airway assessment is mandatory since Fontan patients can have a long history of mechanical ventilation or tracheostomy. Well-planned airway management based on a detailed preoperative airway evaluation is very important in Fontan patients because hypoventilation and subsequent hypercapnia are detrimental via increasing PVR.

In our patient, the nonselective alpha-adrenergic antagonist phenoxybenzamine was administered preoperatively. However doxazosin, a short-acting selective postsynaptic alpha-1 receptor antagonist, might be helpful in patients with pheochromocytoma and single ventricle physiology [6]. Since doxazosin has no 
presynaptic alpha- 2 receptor blocking activity, it might reduce the risk of reflex tachycardia. Furthermore, doxazosin pretreatment might decrease the incidence of longer lasting intraoperative hypotension.

Although there are no established guidelines for postoperative ventilatory management, an early recovery of spontaneous ventilation might be advantageous in patients with a Fontan circulation, because it reduces the peak inspiratory and intrathoracic pressures and subsequently, decreases the PVR [14]. However, postoperative hypoventilation should be avoided, since it results in hypercapnia, hypoxia, and an elevated PVR. Our patient remained temporarily intubated postoperatively due to concern regarding full recovery of consciousness, muscle tone and respiratory function.

In conclusion, laparoscopic excision of a pheochromocytoma can be considered in patients with a Fontan circulation, although this can have many unfavorable effects on hemodynamics. Careful perioperative management with constant vigilance and understanding of the physiology of these patients are essential.

\section{References}

1. Fontan F, Baudet E. Surgical repair of tricuspid atresia. Thorax 1971; 26: 240-8.

2. Ueda T, Oka N, Matsumoto A, Miyazaki H, Ohmura H, Kikuchi T, et al. Pheochromocytoma presenting as recurrent hypotension and syncope. Intern Med 2005; 44: 222-7.

3. Kinney MA, Narr BJ, Warner MA. Perioperative management of pheochromocytoma. J Cardiothorac Vasc Anesth 2002; 16: 359-69.

4. Prys-Roberts C. Phaeochromocytoma--recent progress in its management. Br J Anaesth 2000; 85: 44-57.

5. Kim YH, Oh AY, Kim CS. Anesthetic management of a patient with a singe ventricle and pheochromocytoma. Korean J Anesthesiol 2004; 47: 590-2.

6. Sparks JW, Seefelder C, Shamberger RC, McGowan FX. The perioperative management of a patient with complex single ventricle physiology and pheochromocytoma. Anesth Analg 2005; 100: 972-5.

7. Tjeuw M, Fong J. Anaesthetic management of a patient with a single ventricle and phaeochromocytoma. Anaesth Intensive Care 1990; 18: 567-9.

8. Lovell AT. Anaesthetic implications of grown-up congenital heart disease. Br J Anaesth 2004; 93: 129-39.

9. Fawcett WJ, Haxby EJ, Male DA. Magnesium: physiology and pharmacology. Br J Anaesth 1999; 83: 302-20.

10. James MF, Cronje L. Pheochromocytoma crisis: the use of magnesium sulfate. Anesth Analg 2004; 99: 680-6.

11. Tait AR, Malviya S, Voepel-Lewis T, Munro HM, Seiwert M, Pandit UA. Risk factors for perioperative adverse respiratory events in children with upper respiratory tract infections. Anesthesiology 2001; 95: 299-306.

12. Lemmer JH, Coran AG, Behrendt DM, Heidelberger KP, Stern AM. Liver fibrosis (cardiac cirrhosis) five years after modified Fontan operation for tricuspid atresia. J Thorac Cardiovasc Surg 1983; 86: 757-60.

13. Rosenthal DN, Friedman AH, Kleinman CS, Kopf GS, Rosenfeld LE, Hellenbrand WE. Thromboembolic complications after Fontan operations. Circulation 1995; 92 (9 Suppl): II287-93.

14. Lofland GK. The enhancement of hemodynamic performance in Fontan circulation using pain free spontaneous ventilation. Eur J Cardiothorac Surg 2001; 20: 114-8. 